\title{
Lifetime Optimization for Multi-hop Wireless Sensor Networks with Rate Distortion Constraints
}

\author{
James C. F. Li and Subhrakanti Dey \\ ARC Special Research Centre for Ultra-Broadband Information Networks (CUBIN) \\ Department of Electrical and Electronic Engineering \\ University of Melbourne, Victoria 3010, Australia \\ \{c.li, s.dey@ee.unimelb.edu.au\}
}

\begin{abstract}
In this paper we address a sensor network lifetime optimization problem, where the network is given a task of reconstructing a Gaussian source with a specified rate-distortion constraint. The multiple agents observing the source relay their information through a multihop network to a sink or a base station. Although this problem has been addressed by other authors recently, only upper and lower bounds were obtained to the optimal lifetime as the nonlinear optimization problem was approximated by linearized constraints. We show that by a clever variable substitution, the original nonlinear optimization problem can be reformulated as a convex optimization problem and can be solved by sophisticated convex optimization tools. We provide numerical results comparing our optimal lifetime with the upper and lower bounds obtained in previous work and demonstrate that those bounds are not very tight. Some possible future research directions are mentioned in the concluding remarks. Keywords-Network Lifetime, Wireless Sensor Network (WSN), Rate Distortion, CEO problem.
\end{abstract}

\section{INTRODUCTION}

Wireless sensor networks (WSN) have become a key technology for the 21 st century due to its widespread applications in security, health, disaster response, defense, telecommunications, structural health monitoring etc. These networks usually consist of a collection of sensor nodes connected by wireless communication links. The sensor nodes usually have on-board battery, communication and computation capability. However, due to limited energy resources and a distinct lack of coordination (compared to cellular networks), the usefulness of these networks can become limited unless special care is taken to optimize energy consumption in communication and computation. Optimizing the lifetime of a WSN is thus an important problem. In many typical wireless sensor network applications, a set of nodes or agents measure or collect data from a source or phenomenon of interest (e.g, temperature in a bushfire prone area or surveillance pictures of human movements etc.) and then transmit them over a multi-hop relay network to a sink or a base station where all data are collected and decisions or final estimates are made. In such a network, energy consumption is affected by such diverse parameters as choice of routes, MAC protocols, data rates, transmit power, wireless channel quality and fading etc. Thus,

This work was supported by the Australian Research Council. CUBIN is an affiliated program of National ICT Australia (NICTA). to optimize the lifetime of a WSN, one really has to consider a cross-layer design. This often leads to very complicated mixed-integer nonlinear optimization problems. Some such cross-layer issues with joint power and rate control have been studied in [1], [2]. In particular, [2] studied a joint power and rate control problem for lifetime optimization in a multi-hop wireless sensor network with constraints on outage induced by channel fading. In both [1], [2], non-convex nonlinear optimization problems were transformed into approximate convex optimization problems and solved using sophisticated convex optimization tools.

However, none of these papers considered any specific application task for the WSN under consideration. On the other hand, a recent paper [3] considers lifetime maximization in the context of data distortion. In this paper, the specific task for the WSN is considered to be a remote random source estimation or reconstruction. It is well known that data rates and rate of transmission are intimately related through rate-distortion theory. While higher data rates may allow high quality data reconstruction (e.g, in surveillance camera applications), it may also result in large amount of energy expenditure in a WSN due to multi-hop transmission. This inherent trade-off between transmission rates (to achieve a certain pre-specified distortion threshold) and lifetime of the WSN was studied in [3] for an interference-free WSN where transmission power was kept fixed and the only optimization variables were the data rates in the various links of the WSN. In order to make their nonlinear optimization problem tractable, the authors of [3] made some judicious linear approximations and obtained upper and lower bounds on the optimal network lifetime using linear programming methods.

In our paper, we consider the same problem as studied in [3]. We show that with a proper variable substitution, the nonlinear optimization problem with rate distortion constraints (and the usual flow conservation and energy constraints) can be transformed into a nonlinear but convex optimization problem. With this convex optimization formulation, we use an appropriate Barrier method [4] to solve for the exact optimal lifetime. This optimal lifetime solution is compared with the lower and upper bounds evaluated by the approximations presented in [3] via numerical studies for a 5-node multi-hop network for various distortion thresholds. This convex optimization framework has the potential advantage that more complicated cross-layer optimization problems (with additional constraints 
such as fading related outage constraints as in [2]) can be formulated using the same framework. These sorts of crosslayer issues in WSN lifetime optimization problems with rate-distortion constraints including power and rate control, scheduling, routing etc. will be addressed in future work via this promising nonlinear convex optimization formulation.

\section{System Description}

The WSN we consider in this paper is denoted by a di-graph $\mathcal{G}(\mathcal{V}, \mathcal{L})$, where $\mathcal{L}$ represents the set of all directed links, which are valid when the receiver of the link can interpret correctly the packets sent by the transmitter with maximum power. The set $\mathcal{V}$ comprises of three categories of nodes: $b_{s}$ is the unique base station which collects information from the field and transfers it to end users; $\mathcal{A}$ are the sensor agents observing the phenomenon of interest; set $\mathcal{I}$ are all the intermediate nodes who convey the data generated by sensors to the Base Station $b_{s}$. Since agents could also be relay nodes for other sensors which are closer to the object, the intersection of $\mathcal{A}$ and $\mathcal{I}$ may not be a empty set, but the union of the three is set $\mathcal{V}$.

$$
\mathcal{V}=\left\{b_{s}\right\} \cup \mathcal{A} \cup \mathcal{I}
$$

As defined in [1], three topology matrices $T^{+}, T^{-}$and $T$ are used to described the relationships between sensor nodes and links in this paper.

$$
\begin{gathered}
T_{v l}^{+}= \begin{cases}1 & \text { if } v \text { is the transmitter of link } l \\
0 & \text { otherwise }\end{cases} \\
T_{v l}^{-}= \begin{cases}1 & \text { if } v \text { is the receiver of link } l \\
0 & \text { otherwise }\end{cases} \\
T_{v l}=T_{v l}^{+}-T_{v l}^{-}
\end{gathered}
$$

The energy consumption in this WSN is calculated as follows. All energy consumption is based on the unit of data bits. Within the transmitter, energy is consumed due to data processing at rate $E_{\text {elec }}^{T x}$ and due to the power amplifier at the rate $E_{a m p}^{T x}$. The second part is proportional to $d^{n}$ where $d$ is the distance between the transmitter and the receiver (exponent $n$ is the propagation loss factor which takes different values from between 2 and 6 depending on the terrain). Similarly, $E_{\text {elec }}^{R x}$ denotes the energy consumed for interpreting one bit of data from received packets. Finally, sensor energy cost per bit data sensing is denoted by $E_{\text {sense. }}$ Thus, for a single link, transmit and receive powers and the sensing power at node $v$ are given by

$$
\begin{aligned}
p_{l}^{T x} & =E^{T x} f_{l} \\
& =\left(E_{\text {elec }}^{T x}+E_{\text {amp }}^{T x}\right) f_{l} \\
& =\left(\epsilon_{\text {elec }}^{T x}+\epsilon_{\text {amp }}^{T x} d_{l}^{n}\right) f_{l} \\
p_{l}^{R x} & =E^{R x} f_{l} \\
& =\epsilon_{\text {elec }}^{R x} f_{l} \\
p_{v}^{\text {sense }} & =E^{\text {sense }} R_{v} \\
& =\epsilon^{\text {sense }} R_{v}
\end{aligned}
$$

$\epsilon_{\text {elec }}^{T x}, \epsilon_{a m p}^{T x}, \epsilon_{\text {elec }}^{R x}$ and $\epsilon^{\text {sense }}$ are constant parameters, $f_{l}$ and $R_{v}$ denote the flow rate on the $l$-th link (with distance $d_{l}$ ) and the data rate generated at the $v$-th node respectively.

\section{Problem Formulation AND ITS SOlution}

In this section, we define the lifetime maximization problem as a constrained nonlinear optimization problem, which after a suitable variable substitution, becomes a convex optimization problem. The constraints for this problem are of three types: flow conservation constraints, energy constraints and ratedistortion constraints

\section{A. Flow Constraints}

Define $f$ as the column vector of the flow rates on all links. At any sensor node $v$, the flow conservation constraint is then given by

$$
T_{v \bullet}^{+} \mathbf{f}-T_{v \bullet}^{-} \mathbf{f}=R_{v}
$$

where $T_{v}$ is the $v$-th row of the Topology Matrix. Constraint (4) can appear in different forms when for different types of nodes. As a pure relay node, the right hand side of (4) is zero. Since the base station does not send any information through wireless network, the first part of the left hand side is zero and the summation of the received flows is the same as the sum of data rate generated from all the sensors. These two special cases are then given by the following:

$$
\begin{aligned}
T_{v \bullet}^{+} \mathbf{f}-T_{v \bullet}^{-} \mathbf{f} & =0 \quad \forall v \in \mathcal{I} \backslash(\mathcal{A} \cap \mathcal{I}) \\
-T_{b_{s}}^{-} \bullet & \mathbf{f}=R_{b_{s}}=-\sum_{v \in \mathcal{A}} R_{v}
\end{aligned}
$$

1) Energy Constraints: Define the column vectors of transmit powers and received powers of all links as $\mathbf{p}^{T x}$ and $\mathrm{p}^{R x}$. Define also the column vector with the $l$-th being $d_{l}^{n} f_{l}$ as $\mathrm{d}^{n} \circ \mathbf{f}$. In this paper, we are not concerned with the energy capacity of the base station. Consequently, the power constraint for the sensor nodes is

$$
\left(T_{v \bullet}^{+} \mathbf{p}^{T x}+T_{v}^{-} \mathbf{p}^{R x}+p_{v}^{\text {sense }}\right) L_{t} \leq E_{v}, \quad \forall v \in \mathcal{A}
$$

$L_{t}$ is the lifetime of the network, which is defined as the length of time before the first node runs out of its battery energy. For a relay node, the sensing power is negligible. Associated with (1), (2), (3), the energy constraints are then given by

$$
\begin{gathered}
\left(T_{v \bullet}^{+}\left(\epsilon_{\text {elec }}^{T x} \mathbf{f}+\epsilon_{a m p}^{T x} \mathbf{d}^{n} \circ \mathbf{f}\right)+T_{v \bullet}^{-}\left(\epsilon_{\text {elec }}^{R x} \mathbf{f}\right)\right. \\
\left.+\epsilon^{\text {sense }} \times R_{v}\right) L_{t} \leq E_{v}, \quad \forall v \in \mathcal{A} \\
\left(T_{v \bullet}^{+}\left(\epsilon_{\text {elec }}^{T x} \mathbf{f}+\epsilon_{a m p}^{T x} \mathbf{d}^{n} \circ \mathbf{f}\right)+T_{v \bullet}^{-}\left(\epsilon_{\text {elec }}^{R x} \mathbf{f}\right)\right) L_{t} \\
\leq E_{v}, \quad \forall v \in \mathcal{I} \backslash(\mathcal{A} \cap \mathcal{I})
\end{gathered}
$$

In this work, we do not consider channel fading or interference. Therefore power control is not considered in this paper.

2) Rate Distortion Constraints: In the so-called quadratic Gaussian CEO problem, a Gaussian source, is of interest to a base station or a central office (CEO). The flow rates containing the information about the source go through the intermediate nodes (relays) to the central office. The given information is used to decrease the data distortion of the source in the reconstructed version. Prabhakaran et al. derive the rate 
region with different quality of observations at different data collecting agents in [5].

$$
\begin{gathered}
\mathcal{R}_{D}\left(r_{1}, \ldots, r_{|\mathcal{A}|}\right) \triangleq\left\{\left(R_{1}, \ldots, R_{|\mathcal{A}|}\right):\right. \\
\sum_{k \in \mathcal{W}} R_{k} \geq \sum_{k \in \mathcal{W}} r_{k}+\frac{1}{2} \log \frac{1}{D} \\
-\frac{1}{2} \log \left(\frac{1}{\sigma_{X}^{2}}+\sum_{k \in \mathcal{A} \backslash \mathcal{W}} \frac{1-e^{-2 r_{k}}}{\sigma_{k}^{2}}\right) \\
\forall \mathcal{W} \subseteq \mathcal{A} \quad \mathcal{W} \neq \emptyset\}
\end{gathered}
$$

$\sigma_{X}^{2}$ and $\sigma_{k}^{2}$ are the variance of the source and of the observation at the $k$-th agent observing source $X$, respectively. The distortion threshold $D$ is the maximum allowable distortion we want to guarantee in the reconstructed version. In addition, $r_{1}, \ldots, r_{|\mathcal{A}|} \geq 0$ are auxiliary variables, and

$\mathcal{F}(D) \triangleq\left\{\left(r_{1}, \ldots, r_{|\mathcal{A}|}\right) \in \mathbb{R}_{+}^{|\mathcal{A}|}: \frac{1}{\sigma_{X}^{2}}+\sum_{k=1}^{|\mathcal{A}|} \frac{1-e^{-2 r_{k}}}{\sigma_{k}^{2}} \geq \frac{1}{D}\right\}$

Since $\mathcal{W}$ in (9) is the set of all the nonempty subset of $\mathcal{A}$, the total number of the elements in $\mathcal{W}$ grows exponentially with $|\mathcal{A}|$, more specifically, $2^{|\mathcal{A}|}-1$. This fact dramatically increases the complexity of the algorithm. In order to reduce computational complexity, we only choose agents with higher quality of measurements, i.e. lower measurement noise variance. As in [3], it is assumed that the inaccuracy of the measurement is proportional to the square of the distance between the source and the $k$-th measuring agent:

$$
\sigma_{k}^{2}=\sigma_{0}^{2} d_{k}^{2} \quad \forall k \in \mathcal{A}
$$

On the other hand, the variable quality of the sensors also introduces a trade-off in energy conservation. A node close to the source can represent the source with a lower data rate, but it needs more relay nodes to convey the information to the base station, since it is far away from it. However, if the sensor node is close to the base station, its accuracy in the measurement may be degraded and hence it needs higher rate in order to achieve the same data distortion threshold - $D$. As a result, the energy consumption increases with the rate and the network lifetime is reduced. This trade-off is captured in the following optimization problem.

\section{B. Problem Formulation}

The objective of the optimization problem is to maximize the network lifetime, defined as the time before the first node in the network runs out of its battery energy.

$$
L_{t}=\min _{v \in \mathcal{A} \cup \mathcal{I}} \frac{E_{v}}{P_{v}}
$$

where $P_{v}$ includes transmission power, receiving power, and sensing power in this paper. Associated with the flow constraints (4) (5), energy constraints (7) (8), and rate distortion constraints (9) (10), the constrained optimization problem is

$$
\begin{gathered}
\max _{\mathbf{f}, \mathbf{R}, \mathbf{r}} L_{t} \\
\text { s.t. } \mathbf{f}, \mathbf{R}, \mathbf{r} \geq 0 \\
T_{v \bullet \mathbf{f}}^{+}-T_{v \bullet \mathbf{f}}^{-}=R_{v}, \quad \forall v \in \mathcal{A} \\
T_{v \bullet}^{+} \mathbf{f}-T_{v \bullet}^{-} \mathbf{f}=0, \quad \forall v \in \mathcal{I} \backslash(\mathcal{A} \cap \mathcal{I}) \\
\left(T_{v \bullet}^{+}\left(\epsilon_{\text {elec }}^{T x} \mathbf{f}+\epsilon_{a m p}^{T x} \mathbf{d}^{n} \circ \mathbf{f}\right)+T_{v \bullet}^{-}\left(\epsilon_{\text {elec }}^{R x} \mathbf{f}\right)\right. \\
\left.+\epsilon^{\text {sense }} \times R_{v}\right) L_{t} \leq E_{v}, \quad \forall v \in \mathcal{A} \\
\left(T_{v \bullet}^{+}\left(\epsilon_{\text {elec }}^{T x} \mathbf{f}+\epsilon_{a m p}^{T x} \mathbf{d}^{n} \circ \mathbf{f}\right)+T_{v \bullet}^{-}\left(\epsilon_{\text {elec }}^{R x} \mathbf{f}\right)\right) L_{t} \\
\leq E_{v}, \quad \forall v \in \mathcal{I} \backslash(\mathcal{A} \cap \mathcal{I}) \\
\sum_{k \in \mathcal{W}} R_{k} \geq \sum_{k \in \mathcal{W}} r_{k}+\frac{1}{2} \log \frac{1}{D}-\frac{1}{2} \log \left(\frac{1}{\sigma_{X}^{2}}\right. \\
\left.+\sum_{k \in \mathcal{A} \backslash \mathcal{W}} \frac{1-e^{-2 r_{k}}}{\sigma_{k}^{2}}\right), \quad \forall \mathcal{W} \subseteq \mathcal{A} \mathcal{W} \neq \emptyset \\
\frac{1}{\sigma_{X}^{2}}+\sum_{k=1}^{|\mathcal{A}|} \frac{1-e^{-2 r_{k}}}{\sigma_{k}^{2}} \geq \frac{1}{D}
\end{gathered}
$$

\section{Solutions}

1) Linear Approximation: In [3], Kansal et al give a simple solution to the above optimization problem by replacing the nonlinear constraints with linear ones. As a result, they derived upper and lower bounds of the network lifetime. There are two kinds of variable substitutions in their solution. First, define two variables - $r^{\prime}$ and $r^{\prime \prime}$ - where

$$
0 \leq r^{\prime}=\min _{k \in \mathcal{A}} r_{k} \leq r_{k} \leq \max _{k \in \mathcal{A}}=r^{\prime \prime}
$$

Therefore, (10) can be rewritten as:

$$
\begin{aligned}
& \frac{1}{\sigma_{X}^{2}}+\left(1-e^{-2 r^{\prime}}\right) \sum_{k=1}^{|\mathcal{A}|} \frac{1}{\sigma_{k}^{2}} \geq \frac{1}{D} \\
& \frac{1}{\sigma_{X}^{2}}+\left(1-e^{-2 r^{\prime \prime}}\right) \sum_{k=1}^{|\mathcal{A}|} \frac{1}{\sigma_{k}^{2}} \geq \frac{1}{D}
\end{aligned}
$$

In addition, since

$$
0 \leq 1-e^{-2 r_{k}} \leq 1, \quad \forall k \in \mathcal{A}
$$

the authors of [3] substitute (9) with

$$
\begin{gathered}
\sum_{k \in \mathcal{W}} R_{k} \geq \sum_{k \in \mathcal{W}} r_{k}+\frac{1}{2} \log \frac{1}{D}-\frac{1}{2} \log \left(\frac{1}{\sigma_{X}^{2}}\right) \\
\sum_{k \in \mathcal{W}} R_{k} \geq \sum_{k \in \mathcal{W}} r_{k}+\frac{1}{2} \log \frac{1}{D} \\
-\frac{1}{2} \log \left(\frac{1}{\sigma_{X}^{2}}+\sum_{k \in \mathcal{A} \backslash \mathcal{W}} \frac{1}{\sigma_{k}^{2}}\right)
\end{gathered}
$$

Instead of dealing with the nonlinear constraints, they shrink and expand the feasible area of the original optimization problem by replacing (10) (9) with (13), (15) or with (14), (16) respectively. This allows them to convert the lifetime maximization problem into a linear programming problem which can be solved efficiently. 
2) Convex Optimization: Although Kansal et al derived the upper and lower bound to the original problem, it cannot obtain the exact optimal lifetime, especially when the rate distortion constraint is not severe. The graph in [3] shows that when the normalized distortion threshold increases the gap between the upper and lower bound grows. In this paper, we transform the above optimization problem to a convex optimization problem via a clever variable substitution. Thus, the optimization problem can be solved by sophisticated convex optimization tools. Without making any linear approximations, we solve the original problem directly by introducing vector $\mathrm{U}$. Let

$$
\begin{gathered}
e^{U_{k}} \leq \frac{1}{\sigma_{X}^{2}}+\sum_{i \in \mathcal{A} \backslash \mathcal{W}_{k}} \frac{1-e^{-2 r_{i}}}{\sigma_{i}^{2}} \\
k=1, \ldots, 2^{|\mathcal{A}|}-1
\end{gathered}
$$

and

$$
\begin{gathered}
\sum_{i \in \mathcal{W}_{k}} R_{i} \geq \sum_{i \in \mathcal{W}_{k}} r_{i}+\frac{1}{2} \log \frac{1}{D}-\frac{1}{2} U_{k} \\
k=1, \ldots, 2^{|\mathcal{A}|}-1
\end{gathered}
$$

As long as the inequalities hold for (17) and (18), the original constraints will be satisfied, because of the decreasing property of the function $-\log (\bullet)$. In other word,

$$
\begin{gathered}
\sum_{i \in \mathcal{W}_{k}} R_{i} \geq \sum_{i \in \mathcal{W}_{k}} r_{i}+\frac{1}{2} \log \frac{1}{D}-\frac{1}{2} U_{k} \\
\geq \sum_{i \in \mathcal{W}_{k}} r_{i}+\frac{1}{2} \log \frac{1}{D}-\frac{1}{2} \log \left(\frac{1}{\sigma_{X}^{2}}+\sum_{i \in \mathcal{A} \backslash \mathcal{W}_{k}} \frac{1-e^{-2 r_{i}}}{\sigma_{i}^{2}}\right)
\end{gathered}
$$

Therefore, we can substitute the first group of rate distortion constraints by (17) and (18), which will give an equivalent result for the optimization problem.

Now, instead of maximizing the network lifetime, we try to minimize its inverse $q$ [2]. Accordingly, the reformulated constrained optimization problem can be written as:

$$
\begin{gathered}
\min _{\mathbf{f}, \mathbf{R}, \mathbf{r}, \mathbf{U}} q \\
\text { s.t. } \mathbf{f}, \mathbf{R}, \mathbf{r} \geq 0 \\
T_{v \bullet \mathbf{f}}^{+}-T_{v \bullet}^{-} \mathbf{f}=R_{v}, \quad \forall v \in \mathcal{A} \\
T_{v \bullet}^{+} \mathbf{f}-T_{v \bullet}^{-} \mathbf{f}=0, \quad \forall v \in \mathcal{I} \backslash(\mathcal{A} \cap \mathcal{I}) \\
\left(T_{v \bullet}^{+}\left(\epsilon_{\text {elec }}^{T x} \mathbf{f}+\epsilon_{a m p}^{T x} \mathbf{d}^{n} \circ \mathbf{f}\right)+T_{v \bullet}^{-}\left(\epsilon_{\text {elec }}^{R x} \mathbf{f}\right)\right. \\
\left.+\epsilon^{\text {sense }} \times R_{v}\right)-q E_{v} \leq 0, \quad \forall v \in \mathcal{A} \\
\left(T_{v \bullet}^{+}\left(\epsilon_{\text {elec }}^{T x} \mathbf{f}+\epsilon_{a m p}^{T x} \mathbf{d}^{n} \circ \mathbf{f}\right)+T_{v \bullet}^{-}\left(\epsilon_{\text {elec }}^{R x} \mathbf{f}\right)\right) \\
-q E_{v} \leq 0, \quad \forall v \in \mathcal{I} \backslash(\mathcal{A} \cap \mathcal{I}) \\
\sum_{i \in \mathcal{W}_{k}} r_{i}+\frac{1}{2} \log \frac{1}{D}-\frac{1}{2} U_{k}-\sum_{i \in \mathcal{W}_{k}} R_{i} \leq 0 \\
k=1, \ldots, 2^{|\mathcal{A}|}-1 \\
e^{U_{k}}-\frac{1}{\sigma_{X}^{2}}-\sum_{i \in \mathcal{A} \backslash \mathcal{W}_{k}} \frac{1-e^{-2 r_{i}}}{\sigma_{i}^{2}} \leq 0 \\
k=1, \ldots, 2^{|\mathcal{A}|}-1 \\
\frac{1}{D}-\frac{1}{\sigma_{X}^{2}}-\sum_{k=1}^{|\mathcal{A}|} \frac{1-e^{-2 r_{k}}}{\sigma_{k}^{2}} \leq 0
\end{gathered}
$$

In (24) and (25)

$\mathcal{W}=\left\{\mathcal{W}_{1}, \ldots, \mathcal{W}_{2|\mathcal{A}|-1}:\right.$ all the nonempty subsets of $\left.\mathcal{A}\right\}$

It is clear that this reformulated problem is a convex optimization problem, because:

- (20) and (21) are linear in $\mathbf{f}$;

- (22) and (23) are linear in $f$ and $q$;

- (24) is linear in $\mathbf{R}, \mathbf{r}$, and $\mathbf{U}$;

- (25) is convex in $\mathbf{r}$ and $\mathbf{U}$;

- (26) is convex in $r$.

\section{Numerical Results}

The simulations in this paper are based on a simple case with 5 nodes: nodes $I$ and $I I$ monitor the phenomenon of interest in Source $X$; intermediate nodes $I I I$ and $I V$ are in charge of relaying the information from the sensors to the base station $B S$. Totally, there are 8 links in the network. Apart from taking advantage of relay nodes, sensors $I$ and $I I$ can also transmit data flow to the base station directly by links 7 and 8 . The coordinates for each node (including the position of the source) are shown in Figure 1.

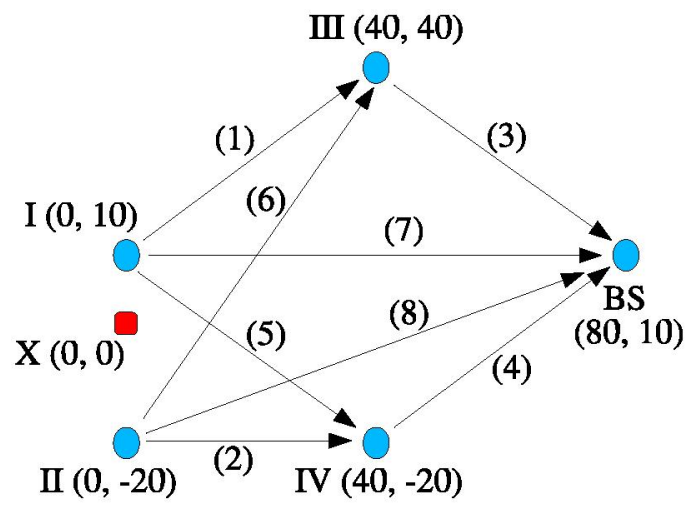

Fig. 1. A Monitoring Instance with 5 Nodes and 8 Links

As mentioned in [6], the energy consumption parameters $\epsilon_{\text {elec }}^{T x}, \epsilon_{a m p}^{T x}, \epsilon_{\text {elec }}^{R x}$ and $\epsilon^{\text {sense }}$ are chosen as $45 \times 10^{-9}$, $10 \times 10^{-12}, 50 \times 10^{-9}$, and $135 \times 10^{-9}$ respectively. The propagation loss factor $n$ is equal to 2. For all sensing agents and relay nodes, the battery capacity is $180 \mathrm{~nJ}$. The inaccuracy in the sensor measurements is given by formula (11). We also suppose $\sigma_{0}^{2}=0.01$ as it is in [3].

In Part III-C.2, we mentioned that $\mathcal{W}$ is the set of all the nonempty subsets of $\mathcal{A}$. For this specific case, $\mathcal{A}=\{I, I I\}$,

$$
\mathcal{W}=\{\{I\},\{I I\},\{I, I I\}\}
$$

We use the classic MATLAB toolbox and the well-known Barrier Method with a logarithmic barrier function [4] to solve the nonlinear convex optimization problem. Equality constraints (20) (21) are eliminated through linear variable substitution. The objective function increases by multiplying $10(\mu$ in [4]) in each round and the back tracking parameters are chosen as $\alpha=0.1 \beta=0.75$ which can be different without losing the convergence. 


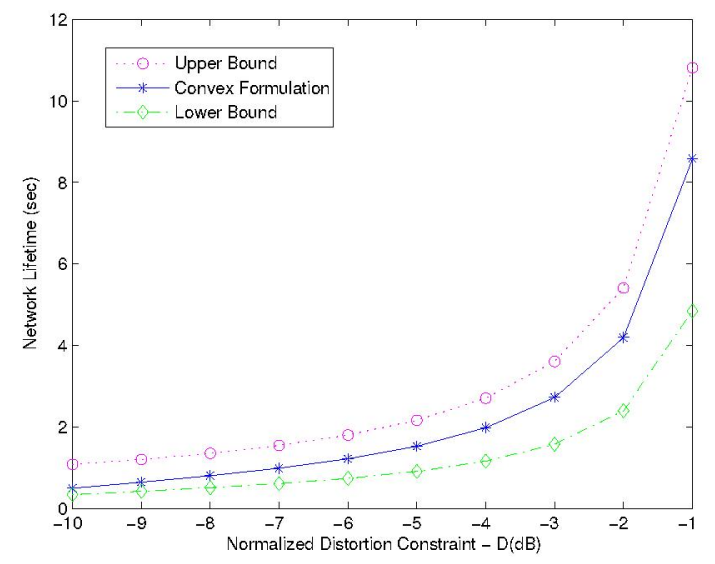

Fig. 2. Compare of Convex Optimization and Linear Approximation

The $x$-axis in Figure 2 represents the rate distortion threshold which is log-normalized by the variance of the source - $\sigma_{X}^{2}$. The graph shows that our optimal lifetime solution sits in between than the upper and lower bounds illustrated by Kansal et al (as expected). It also clearly shows that none of the bounds are particularly tight. The differences among the three lifetime solutions are considerable when the rate distortion threshold is not too demanding. The network lifetime becomes very large when the logarithmic distortion threshold approaches 0 . This fact can be as follows: if $D=$ $\sigma_{X}^{2}$, we can simply estimate the source by the mean value of the source (this information can be received by communication overhead), and this guess will achieve the required distortion threshold; in other words, the estimator at the base station can satisfy the requirement without receiving any information from the sensors at all. Therefore, no transmission is necessary, the energy consumption is zero, and then the lifetime goes to infinity. On the other hand, if the rate distortion constraint is too demanding, the optimization problem can become infeasible. The network lifetime becomes zero in that case. Figures 3 and 4 give the complete solution for routing and rate allocation when $D=-1(\mathrm{~dB})$ and $D=-10(\mathrm{~dB})$ respectively. It is clear from the graphs that the data rate $R_{1}, R_{2}$ for the stricter constraint $D=-10(\mathrm{~dB})$ are much greater than those for $D=-1(\mathrm{~dB})$.

\section{CONCLUSION AND FUTURE WORK}

This convex optimization framework is extremely useful as it allows us to consider other constraints in the optimization problem imposed by other layers in the network, such as rate outage and link outage constraints as in [2] and scheduling or routing constraints etc. In future work, we will study such cross-layer issues for sensor networks with rate-distortion constraints as well as data rate constraints when the task of the network is not just limited to reconstructing a random source, but the network is responsible for estimating a complex dynamical system (e.g, the trajectory of a moving target).

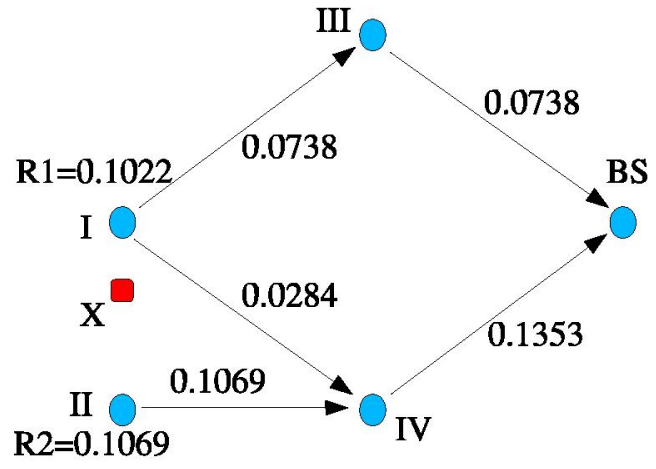

Fig. 3. Solution (Unit: bps) to the Convex Optimization when $D=-1(\mathrm{~dB})$

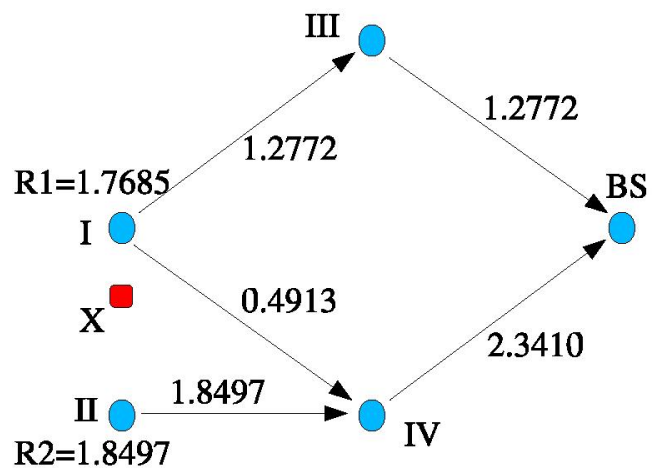

Fig. 4. Solution (Unit: bps) to the Convex Optimization when $D=-10(\mathrm{~dB})$

\section{REFERENCES}

[1] R. Madan, S. Cui, S. Lall, and A. Goldsmith, "Cross-layer design for lifetime maximization in interference-limited wireless sensor networks," in IEEE INFOCOM' 05 , vol. 3, 2005, pp. 1964-1975.

[2] J. C. F. Li and S. Dey, "Lifetime optimization for wireless sensor networks with outage probability constraints," in Proc. European Wireless 2006 , April 2006, to appear.

[3] A. Kansal, A. Ramamoorthy, M. B. Srivastava, and G. J. Pottie, "On sensor network lifetime and data distortion," in IEEE ISIT '05, 2005, pp. $6-10$.

[4] S. Boyd and L. Vandenberghe, Comex Optimization. Cambridge University Press, 2004.

[5] V. Prabhakaran, D. Tse, and K. Ramchandran, "Rate region of the quadratic gaussian CEO problem," in IEEE ISIT '04, 2004, p. 119.

[6] W. Heinzelman, "Application-specific protocol architectures for wireless networks," Ph.D. dissertation, Massachusetts Institute of Technology, 2000.

[7] T. S. Rappaport, Wireless Communications: Principles and Practice. Prentice Hall, 1996. 